\title{
Distribution Network Communication Network Hybrid Network Technology Research
}

\author{
Xin $\mathrm{Xu}^{1}$, Haijun Ren ${ }^{2}$, Ruilin $\mathrm{Xu}^{3}$, Lan Long ${ }^{2}$ \\ ${ }^{1}$ Chongqing State Grid Electric Power Research Institute, China \\ ${ }^{2}$ The School of Software Engineering Chongqing University, China \\ ${ }^{3}$ Chongqing State Grid Electric Power Research Institute, China
}

\begin{abstract}
Keywords: Power grid communication system, Hybrid networking system, Communication network.
\end{abstract}

\begin{abstract}
Synthetically analyze the power distribution communication network technology in the smart power grid link. It mainly includes the summary of the smart distribution network, electric power communication backbone network and then layer communication requirements, the network mode and the application mode of WLAN technology in the distribution network, EPON network technology, micropower wireless technology, PTN technology and power broadband wireless systems. State the demand of the smart power distribution business and put forward technical guidance for the smart power distribution communication network scheme. In view of the complex distribution network architecture, more nodes and scattered, complex business types data business needs, put forward the integrated solutions of optical fiber communication as the backbone access, wireless bandwidth, passive optical communication as the coverage, the short distance wireless communication as the main terminal access. It provides a reference for the communication plan of distribution side.
\end{abstract}

\section{Power Grid Communication System Structure}

The Smart Power Grid Communication Needs. The smart grid is the real-time monitor to the critical equipment running status such as power generation, transmission, distribution, power supply with sensors. Then collect and integrate the obtained data through the network system. At last, analyze and excavate the data in order to realize the optimal management of the whole power system operation. Smart grid has the self-healing, interactive, optimization, compatible and integrated feature. Realize information and interaction between the grid system equipment. Therefore, upload information and instructions need a reliable, safe, high speed electric power communication network to support. The smart grid construction involve power generation, transmission, substation, power distribution and utilization. In the side of electricity, it will use the advanced measurement, effective control, high-speed communication, fast energy storage technologies. Realize the new type of power supply. The new type of power supply is quick response, accurate measurement, data real-time, charge diversity, convenient services, power flow, information flow, business flow of real-time interaction. In order to adapt the requirements of the smart grid management, information transmission and exchange, communication network need further promotion in the transmission rate, reliability, and security, etc. Built into the next generation optical transmission network which has the ability of time synchronization and service awareness, the large capacity, high speed, real time. The smart grid construction is a huge system project. The future power grid interconnection and two-way communication need standard interoperability framework to support.

Our Country Electric Power Communication Network Structure. Our country electric power communication network structure is a hierarchical design. It can be divided into backbone layer, convergence layer and access layer. Backbone layer mainly refers to a national network and network performance among provinces. It includes the backbone transmission network and city core network. It covers power generation, transmission, substation, etc. Convergence layer mainly refers to the network in the province and local network. The access layer mainly refers to business terminal access networks. It is the communication network extension of power supply company backbone around the city. It mainly covers the distribution and utilization. 
The Composition of Distribution Automation System. Distribution automation system use a variety of communication modes. It can realize data acquisition, remote control. So as to fast remove fault node and restore power automatically. Distribution automation system is usually composed of power distribution master station, power distribution terminal/electronic and communication network three parts. Master station system realize distribution scheduling, production and operation with the superior dispatching automation system, geographical information system, and other external systems relation. Distribution master station mainly includes the distribution SCADA and the grid analysis applications. It has the function of data acquisition, status monitoring, remote control, alarm, accident recall events, etc. Grid analysis is responsible for splicing, topology analysis, load prediction, early warning analysis, etc. Electronic station is responsible for power distribution terminal data acquisition and forwarding within the area. They are usually placed in a substation or switching station. Power distribution terminal mainly refers to remote terminal installed in distribution facilities such as a switching station, substation, ring network cabinet, ect. The distribution automation system structure is shown in Fig 1.

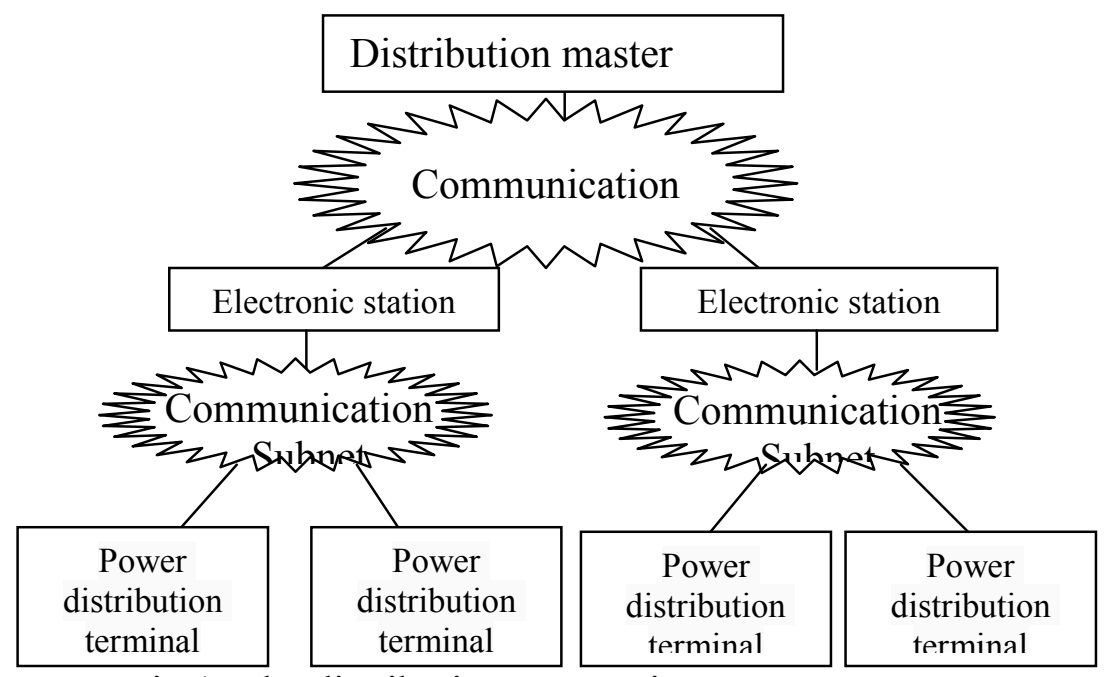

Fig 1: The distribution automation system structure

\section{The Main Technology in Hybrid Networking System}

EPON Technology. PON technology is an advanced communication technology. With the development of optical fiber communication technology, Optical fiber and optical fiber equipment costs are reduced. PON technology is mainly divided into three kinds, APON, EPON and GPON. EPON is PON network based on Ethernet protocol. EPON system mainly consists of three parts. They are the optical line terminal, optical distribution network and the optical network unit. It is shown in Fig 2.

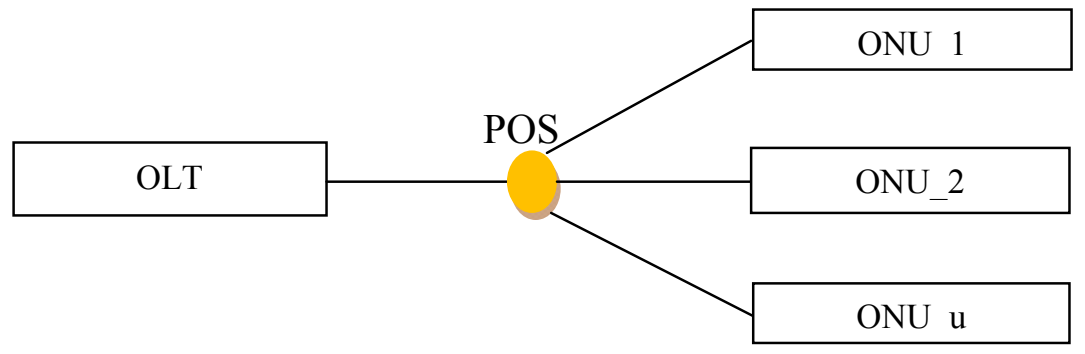

Fig 2: System components of EPON

OLT is the passive optical network local side equipment. It is the core system in the EPON. It's usually placed in the room. It is a platform with many business and can support IP and traditional TDM business. It provides a variety of business interface. ODN is mainly composed of passive divider and optical fiber. POS connects each ONU equipment through shunt the light. Complete the downstream data distribution and uplink data set. POS is usually an even number of spectral. The 
specific number of spectral need consider the actual requirement. POS is a passive device, it can place need position in line. The ONU is in the user side of EPON system. It is used to connect the user terminal equipment. It can connect electric power equipment such as RTU, concentrator and so on in the electrical system .ONU uplink interface is PON. It connects passive optical network through the optical fiber. Its downward interface is rich.

Working Principle of the EPON. EPON is point to multipoint system structure. It uses multi-point control protocol. Its working principle is as follows. After OLT equipment electric start in the local side, first, periodic broadcast allows access time slot information on the PON port. According to the allows access time slot information, ONU sends registration request. When the OLT receive the request, authenticate ONU. If the certification pass, allow them to access. ONU is assigned the only logical link id.

\section{Hybrid Network Communication Project Design}

The Overall Topology of Design Scheme.Hybrid network design is in the basis of the passive optical network solutions and industrial switch solution. The overall plan and design the network equipment hardware and software aspects to implement. It puts the two current independent operation access network together. So it makes the network design and project implementation more flexible. In the network level, it can realize the better optimization. Provide another access mode for complex access network.

Design Scheme Detailed Description. In the hybrid network scheme design, Industrial switches or OLT equipment can connect with electronic station switches through multiple ports of fiber link. Using the Ethernet link aggregation technology, increase the transmission bandwidth between access network and the core layer. Industrial switches and OLT equipment linked port is usually a gigabit speeds. With the increase of quantities of data terminal with power grid and the future new business applications, gigabit linked rate is not meet and guarantee the safe operation of the business. So bundle more than one gigabit port into a single logical interface, this part can increase the transmission bandwidth. With the maturity of the future higher transmission bandwidth technology and cost down, industrial switches and OLT equipment linked transmission bandwidth can directly upgrade to the Wan Zhao above. The link aggregation techniques can be applied to the more lower level access network. At the same time, using the link aggregation technology, also can improve the safety of the industrial switches and OLT equipment top allied interface. As one of the top allied interfaces failure and can not transmit data through the link, the power grid terminal data can still be transmitted by other polymerized top allied interface. Ensure the power system business real-time and continuity and avoid electricity accidents due to communication problems. After the failure of the top allied interface restores, they can be added to the link aggregation group automatically and begin to transmit data flow. In the distribution automation and electricity information collection system, distribution master station connect through SDH equipment and various electronic stations.

\section{Design Scheme Analysis.}

a)Inherit the characteristics of passive optical network solutions and industrial switches solution. Such as the optical fiber communication has high bandwidth transmission ability, strong anti-interference, good secrecy, etc. Still using the mature Ethernet technology makes the compatibility and stability of the communication system guaranteed. And expansion of communication system is still very convenient and the construction cost is still very low. At the same time, the passive optical network hand in hand ring network protection and industrial switch Ethernet ring network model also can be used in hybrid network system. The mixing network system security can still be secure.

b)Hybrid network design scheme can optimize grid access network level. In the original plan, passive optical network system and industrial switches system is generally independently run. Each 
the underlying terminal data communication or cooperative work need to upload the data to the respective electronic station. And then sent the data to the terminal through the purpose electronic station. Even though the system has intersection operation, mostly also put a system hook up to a lower another system. The system is another system's access layer. From the network structure, not only increases the communication level, but also increase the communication nodes. The increase of the communications node is bound to increase the probability of failure occurs. It generates a certain influence on the stability of the communication network. Mixed network design is to meet grid data terminal access, at the same time, optimizes the access network structure and avoid complex network topology.

c)Increases the original distribution automation and information acquisition system access network expansion mode. If adopt the industrial switches construction communication access network, usually will adopt industrial switches in the network expansion. Faced with the decentralized communication node such as switching station or switching room, it needs more deployment industry switches. If each node deploy an industrial switches, each node is connected with a few of data terminal. So if a one-time deploy multiport switch, the cost of investment will increase and extra port need not wasteful. If the switches have few deployment port, expansion in the future will be inconvenient.

d)Increase the system access network protection mode. Industrial switches solutions realize the protection of data terminal through the Ethernet ring or more Ethernet subnet. Passive optical network solutions build hand in hand model to protect data terminal access between the two electronic stations. Using hybrid network design, we can establish hand in hand model data terminal protection under the Ethernet ring. Put the hand in hand protection down on access layer, so enrich the original Ethernet protected mode.

\section{Conclusion}

With the arrival of the smart grid construction boom around the world and more and more innovative technology application in the construction of smart grid. It is good for new energy and renewable energy development and utilization. In the new energy development planning of our country, due to the power grid construction lag, it has the influence on the new energy. So it should cover new energy in the power grid planning, prevent the development of power and power grid planning disconnected. At present, the smart grid construction in China proceed according to the phase step. In the process of power distribution and power consumption, they have launched some new and renovation project around the grid company.

\section{Acknowledgement}

The paper is supported by the Follows Projects:

(1) No.CDJZR12090004 supported by the Fundamental Research Funds for the Central Universities

(2) No.CDJZR1309551 supported by the Fundamental Research Funds for the Central Universities

\section{References}

[1] Kramer, G. Pesavento, G. Ethernet passive optical network (EPON): building a next-generation optical access network[J]. IEEE Communications Magazine,2002(2):66-73.

[2] G Kramer, B Mukherjee, G Pesavento. IPACT: A dynamic protocol for an Ethernet PON(EPON)[J]. IEEE Communications Magazine, 2002.40 (2):74-80. 